controls which allele is expressed. Second, DNA methylation can recruit methylated $\mathrm{CpG}^{-}$binding proteins, which progressively induce a heterochromatic state that inhibits transcription ${ }^{4}$. However, both knock-in alleles are highly transcribed ${ }^{3}$, challenging the idea that DNA methylation acts in this way. Nevertheless, transcription from heterochromatin can occur; thus, it would be useful to determine the nuclear localization of the two knock-in alleles relative to that of heterochromatin clusters. An alternative possibility is that DNA methylation inhibits an SHM-specific set of histone modifications, such as phosphorylation of histone H2B at Ser14 (ref. 2), distinct from those that govern transcription. It will be useful to explore the association of such histone modifications with methylated versus demethylated alleles in the knock-in model. Such studies might also help to explain why the methylated alleles can still be transcribed.

This raises the central question of this topic. How important is inhibition of SHM by DNA methylation in the normal situation? In this case, VJ-recombined alleles, whether productive or nonproductive, are always demethylated and transcribed and always undergo SHM; thus, only the germline allele remains methylated and fails to undergo SHM. Is the germline allele further modified to become heterochromatin in cells undergoing SHM? Association of immunoglobulin alleles with heterochromatin after allelic exclusion is relaxed in resting splenic B cells, but one allele seems to be repositioned at centromeric heterochromatin in activated B cells ${ }^{11}$. Such repositioning by itself might prevent SHM. However, both mechanisms may be nonredundantly required, particularly if DNA methylation inhibits a different process, such as SHM-specific histone modification. Furthermore, as SHM is reduced to approximately $10 \%$ but is not completely inhibited by DNA methylation, heterochromatin modification may be an extra safeguard of genomic integrity. More detailed analyses of nuclear localization and histone modification of immunoglobulin alleles in purified populations undergoing SHM will distinguish these possibilities.

This study generates an intriguing puzzle. SHM is detrimental to genomic integrity, so why is the nonproductive allele not remethylated? It seems the $B$ cell is paying a heavy price for its second chance at $\mathrm{V}(\mathrm{D}) \mathrm{J}$ recombination. But perhaps it has no alternative. If a putative element that controls DNA methylation is present in the region between the $\mathrm{V}$ and $\mathrm{J}$ genes, this element would be lost by the process of V-J recombination. This region already contains an element involved in the recruitment of Igk alleles to heterochromatin ${ }^{12}$ and warrants investigation for other regulatory elements.

The study here by Fraenkel et al. ${ }^{3}$ has important implications for the immunoglobulin heavy chain, both for SHM and for class-switch recombination, a second antibody-diversification process driven by activa- tion-induced cytidine deaminase. It raises the possibility of an additional difference between the SHM of the gene encoding immunoglobulin heavy chain $(I g h)$ and the SHM of $I g k$, as differential demethylation has not been shown to be involved in $\mathrm{V}(\mathrm{D}) \mathrm{J}$ recombination and allelic exclusion of $I g h$ and indeed V(D)J recombination of Igh can occur on methylated DNA ${ }^{13}$. Further studies of the Igh locus are needed; it would also be useful to study SHM in models in which two pre-rearranged heavy chains are expressed ${ }^{7,8}$.

\section{COMPETING INTERESTS STATEMENT}

The authors declare no competing financial interests.

1. Di Noia, J.M. \& Neuberger, M.S. Annu. Rev. Biochem. advance online publication 28 February 2007 (doi: 2010.1146/annurev. biochem.2076.061705.090740)

2. Odegard, V.H. \& Schatz, D.G. Nat. Rev. Immunol. 6 573-583 (2006).

3. Fraenkel, S. et al. Nat. Immunol. 8, 715-722 (2007).

4. Klose, R.J. \& Bird, A.P. Trends Biochem. Sci. 31 89-97 (2006).

5. Corcoran, A.E. Semin. Immunol. 17, 141-154 (2005).

6. Delpy, L., Sirac, C., Le Morvan, C. \& Cogne, M J. Immunol. 173, 1842-1848 (2004).

7. Gerdes, T. \& Wabl, M. Nat. Immunol. 5, 1282-1287 (2004).

8. Sonoda, E. et al. Immunity 6, 225-233 (1997).

9. Jolly, C.J. \& Neuberger, M.S. Immunol. Cell Biol. 79 , 18-22 (2001).

10. Larijani, M. et al. Mol. Immunol. 42, 599-604 (2005).

11. Skok, J.A. et al. Nat. Immunol. 2, 848-854 (2001).

12. Liu, Z. et al. Immunity 24, 405-415 (2006).

13. Johnson, K. et al. Nat. Immunol. 5, 853-861 (2004).

\title{
Armed and ready: How effector T cells deploy in reactive lymph nodes to modulate immunity
}

\author{
David W O’Neill \& Nina Bhardwaj
}

\section{New evidence demonstrates how two different effector $T$ cell subsets traffic in reactive lymph nodes to modulate $T$ cell and $B$ cell responses.}

\begin{abstract}
Tcells home to specific locations in secondary lymphoid tissue by means of their differential expression of chemokine receptors. For example, naive $\mathrm{T}$ cells and central memory $\mathrm{T}$ cells ( $\mathrm{T}_{\mathrm{CM}}$ cells) home to $\mathrm{T}$ cell areas of lymphoid tissue through their expression of CCR7, whereas effector $\mathrm{T}$ cells ( $\mathrm{T}_{\mathrm{E}}$ cells) and effector
\end{abstract}

David W. O'Neill and Nina Bhardwaj are with the New York University School of Medicine, New York, New York 10016, USA.

e-mail: nina.bhardwaj@med.nyu.edu memory $\mathrm{T}$ cells ( $\mathrm{T}_{\mathrm{EM}}$ cells) lose expression of CCR7 and traffic in peripheral tissues $\left(\mathrm{T}_{\mathrm{CM}}\right.$ cells and $\mathrm{T}_{\mathrm{EM}}$ cells are subsets of memory $\mathrm{T}$ cells defined by the absence or presence, respectively, of immediate effector function and the ability to home to peripheral rather than lymphoid tissue $)^{1}$. In this issue of Nature Immunology, two papers provide new evidence that $\mathrm{T}$ cell subsets can traffic in reactive lymph nodes through expression of the chemokine receptors CXCR3 and CXCR5 to inhibit or enhance immune responses. Findings from both papers have implications for vaccine development, administration and monitoring.

In the first paper, Guarda et al. ${ }^{2}$ show how $\mathrm{CD}^{+} \mathrm{T}_{\mathrm{E}}$ and $\mathrm{T}_{\mathrm{EM}}$ cells, which express CXCR3, home to high endothelial venules (HEVs) in reactive but not resting lymphoid tissue through interactions with inducible CXCR3 ligands in the HEV lumen. These cells then gain entry to $T$ cell areas of the reactive node, where they can target and kill cognate antigen-bearing dendritic cells (DCs) as a means to limit secondary, and perhaps even primary, immune responses. In the 


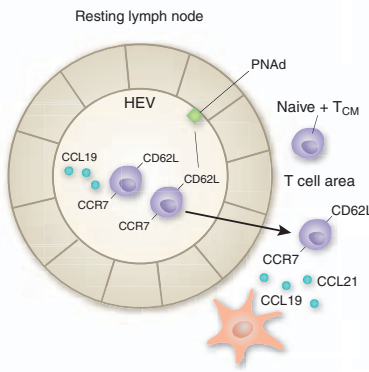

b

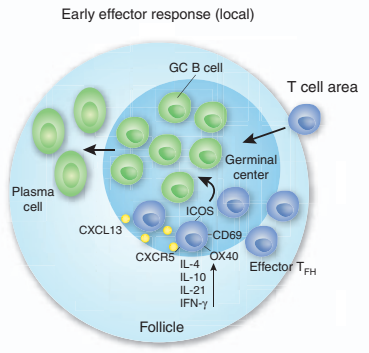

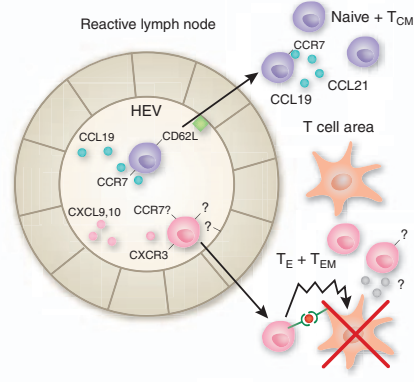

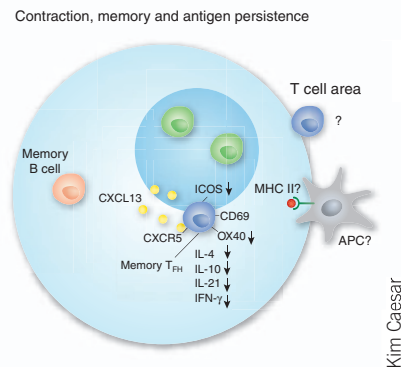

Figure 1 Effector T cell subsets traffic in T cell and B cell areas of lymph nodes to modulate immune responses. (a) Naive $C D 8^{+} T$ cells and $T_{C M}$ cells home to HEVs and T cell areas in secondary lymphoid tissue through their expression of L-selectin and CCR7 (left). In contrast, CD8 ${ }^{+} \mathrm{T}_{\mathrm{E}}$ and $\mathrm{T}_{\mathrm{EM}}$ cells lack L-selectin and CCR7 and do not migrate toward resting nodes. However, $\mathrm{T}_{E}$ and $\mathrm{T}_{\mathrm{EM}}$ cells have higher expression of CXCR3 and migrate to HEVs in reactive lymph nodes (right), which upregulate expression of CXCR3 ligands (CXCL9 and CXCL10). $\mathrm{T}_{E}$ and $\mathrm{T}_{\mathrm{EM}}$ cells then gain access to $T$ cell areas of the node through interactions with unknown adhesion molecules and chemokines ('?'), where they can kill cognate DCs to limit secondary immune responses. PNAd, peripheral node addressin. (b) $\mathrm{T}_{\mathrm{FH}}$ cells are specialized $\mathrm{CD} 4^{+} \mathrm{T}$ cells that express CXCR5 and home to B cell follicles through interactions with CXCL13. In the effector phase (left), $\mathrm{T}_{\mathrm{FH}}$ cells accumulate in lymphoid tissue draining sites of inoculation and express molecules that promote the generation of germinal center (GC) B cells and plasma cells. With contraction of the $\mathrm{B}$ cell response (right), the number of $\mathrm{T}_{\mathrm{FH}}$ cells decreases, although 'reservoirs' of antigen-specific $\mathrm{T}_{\mathrm{FH}}$ cells persist in conjunction with antigen-MHC class II. The persistent $\mathrm{T}_{\mathrm{FH}}$ cells are $\mathrm{ICOS}^{10} \mathrm{OX} 4 \mathrm{O}^{\mathrm{lo}}$ and have less expression of effector cytokines, but they express CD69 and have recall response ability. Persistent antigen-MHC class II may be provided by dendritic cells (which can be found in B cell zones ${ }^{12}$ ), although the precise cell type is not known ('?'). IL-, interleukin; IFN- $\gamma$, interferon- $\gamma$; MHC II, MHC class II; APC, antigen-presenting cell. second paper, Fazilleau et al. ${ }^{3}$ focus on follicular $B$ helper T cells ( $\mathrm{T}_{\mathrm{FH}}$ cells), a subset of antigenexperienced $\mathrm{CD} 4^{+} \mathrm{T}$ cells known to traffic to $B$ cell follicles through expression of CXCR5 to promote antibody responses ${ }^{4}$. The authors demonstrate that 'effector' $\mathrm{T}_{\mathrm{FH}}$ cells accumulate 'preferentially' in lymphoid tissue draining sites of vaccination, locally promoting germinal center formation and the generation of plasma cells. As the antibody response contracts, $\mathrm{T}_{\mathrm{FH}}$ cells diminish in number and function but persist at the site. They retain the ability to mount effector function after local re-exposure to antigen, thus demonstrating a 'memory' $\mathrm{T}_{\mathrm{FH}}$ phenotype.

In the steady state, naive $\mathrm{T}$ cells and $\mathrm{T}_{\mathrm{CM}}$ cells home to HEVs and $\mathrm{T}$ cell areas in secondary lymphoid tissue through their expression of L-selectin (CD62L), the integrin LFA-1 and the receptor CCR7, which interact with L-selectin ligands (peripheral node addressin), intercellular adhesion molecules and the chemokines CCL19 and CCL21 (Fig. 1a). In contrast, CD8 ${ }^{+}$ $\mathrm{T}_{\mathrm{E}}$ and $\mathrm{T}_{\mathrm{EM}}$ cells lack L-selectin and CCR7, have higher expression of CXCR3 and traffic in tissues, blood and spleen. They also acquire the ability to bind P- and E-selectin, which along with CXCR3 expression permits homing to inflamed tissues where the effector cells lyse targets bearing cognate antigen. Guarda et al. ${ }^{2}$ present the new observation that $\mathrm{CD} 8^{+} \mathrm{T}_{\mathrm{E}}$ and $\mathrm{T}_{\mathrm{EM}}$ cells, which are generally believed to avoid lymph nodes (although this has been described for infection with human immunodeficiency virus ${ }^{5}$ ), can in fact migrate to lymph nodes but 'preferentially' traffic to reactive rather than resting nodes. They obtained this result in reactive nodes conditioned by a variety of stimuli, including mature DCs, tumor necrosis factor, vaccine adjuvant and local listeria infection.
Guarda et al. ${ }^{2}$ show that $\mathrm{CD}^{+} \mathrm{T}_{\mathrm{E}}$ and $\mathrm{T}_{\mathrm{EM}}$ cells rapidly migrate to and re-enter reactive lymph nodes through HEVs by a mechanism that depends mainly on expression of CXCR3, which interacts with HEV-lumenal CXCR3 ligands (CXCL9 and CXCL10; Fig. 1a). Notably, they show that these ligands are rapidly induced in the HEV lumen in reactive nodes by inflammatory stimuli but are not expressed in resting lymph node HEVs. Migration of $\mathrm{T}_{\mathrm{E}}$ and $\mathrm{T}_{\mathrm{EM}}$ cells to reactive lymph nodes may depend partly on low expression of CCR7 on the T cells, as T cells deficient in both CCR7 and CXCR3 were less able to home to reactive lymph nodes than were T cells deficient in CXCR3 alone. The authors report that after gaining entry to the node, $\mathrm{CD}^{+} \mathrm{T}_{\mathrm{E}}$ and $\mathrm{T}_{\mathrm{EM}}$ cells limit the induction of secondary responses by killing DCs bearing cognate antigen ${ }^{2}$. This is the first time, to our knowledge, that T cell-mediated killing of cognate DCs has been demonstrated in lymphoid tissue (it has been reported in peripheral tissues before $)^{6}$.

$\mathrm{T}_{\mathrm{FH}}$ cells are a specialized type of antigenexperienced $\left(\mathrm{CD} 45 \mathrm{RO}^{+}\right) \mathrm{CD}^{+}$helper $\mathrm{T}$ cell that orchestrates $\mathrm{B}$ cell responses in secondary lymphoid tissues. These cells home to B cell follicles through interactions between CXCR5 and CXCL13 (CXCL13 is produced by stromal cells in B cell follicles, primed $\mathrm{T}_{\mathrm{FH}}$ cells, and myeloid and plasmacytoid DCs) ${ }^{4} \cdot \mathrm{T}_{\mathrm{FH}}$ cells promote $\mathrm{B}$ cell responses, including $\mathrm{B}$ cell memory development and plasma cell differentiation, through their expression of costimulatory molecules and cytokines such as ICOS, OX40, CD40L and interleukins 10 and 21 (ref. 4). How these cells develop and whether they have the capacity for memory are issues now being investigated.
The paper by Fazilleau et al. $^{3}$ sheds light on both issues. The authors report local accumulation of $\mathrm{T}_{\mathrm{FH}}$ cells after vaccination with a foreign protein (pigeon cytochrome $c$ ) using two different adjuvants. Subcutaneous vaccination promotes high-affinity helper $\mathrm{T}$ cell responses in draining lymph nodes but promotes them to a much lesser extent in spleen. A substantial fraction of these helper T cells are $\mathrm{CXCR}^{+} \mathrm{ICOS}^{+} \mathrm{OX} 40^{+}$effector $\mathrm{T}_{\mathrm{FH}}$ cells (Fig. 1b). The development of these cells is associated with the production of germinal center B cells and plasma cells. Notably, the reverse is true with intraperitoneal vaccine administration: highaffinity $\mathrm{T}_{\mathrm{FH}}$ cells, germinal center $\mathrm{B}$ cells and plasma cells develop in the spleen but develop to a much lesser extent in lymph nodes.

After contraction of the B cell response, the authors report fewer $\mathrm{T}_{\mathrm{FH}}$ cells in the draining node ${ }^{3}$. However, 'reservoirs' of antigen-specific $\mathrm{T}_{\mathrm{FH}}$ cells persist in the node for over 28 days. These persistent $\mathrm{T}_{\mathrm{FH}}$ cells are $\mathrm{ICOS}^{\mathrm{lo}} \mathrm{OX} 40^{\mathrm{lo}}$ and have less effector capacity and lower expression of genes encoding effector cytokines (Fig. 1b). However, they have recall response ability, regaining expression of effector cytokine mRNA after antigen re-exposure. In association with this 'memory' $\mathrm{T}_{\mathrm{FH}}$ cell compartment, the authors note the development of antigen-specific memory B cells and, by indirect evidence, the presence of persistent complexes of peptide and major histocompatibility complex (MHC) class II that they detected over 70 days after priming (although they do not identify a specific cell responsible for this). In addition, they detected continued expression of CD69 in a subset of these memory $\mathrm{T}_{\mathrm{FH}}$ cells as late as 56 days after priming. They suggest that CD69, which may locally tether the lymphocytes through 
ligation of an as-yet-undescribed ligand, as well as the persistence of specific antigen, help to maintain the memory $\mathrm{T}_{\mathrm{FH}}$ cell population in the draining node ${ }^{7,8}$.

The results of these studies raise many issues. Although CXCR3 ligands promote the homing of $\mathrm{CD}^{+} \mathrm{T}_{\mathrm{E}}$ and $\mathrm{T}_{\mathrm{EM}}$ cells to HEVs in reactive nodes $^{2}$, it is still not apparent what molecular interactions are involved in the sticking and rolling of these cells on the HEV lumen and their migration into T cell area of the node (Fig. 1b). In addition, it is not known how long the $\mathrm{CD} 8^{+}$ effector $\mathrm{T}$ cells persist in reactive lymph nodes. Also, the homing of effector $\mathrm{CD} 4^{+} \mathrm{T}$ cells, which apparently traffic by different mechanisms, is not addressed in these studies.

One chief unresolved issue is the relative importance of the phenomenon of DC killing in the node by cognate $\mathrm{CD}^{+}$effector T cells. Is this an important means of negative feedback? In addition, what is the main site of DC elimination by effector $\mathrm{CD} 8^{+} \mathrm{T}$ cells; is this reactive lymphoid tissue or the periphery? A published study has reported that DC elimination by this mechanism occurs in the periphery but not in lymph nodes ${ }^{6}$.

Another important issue is how to reconcile the observed killing of cognate DCs in reactive nodes with the established effectiveness of many booster vaccination regimens ${ }^{9}$. What makes some DCs stimulatory, whereas some DCs apparently become targets? One possible expla- nation is that DCs can be rendered resistant to killing by cytolytic T lymphocytes depending on the maturation stimulus. A published study has shown that DCs activated by lipopolysaccharide, CD40L or Thelper type $1 \mathrm{CD} 4^{+} \mathrm{T}$ cells (but not those activated by $\mathrm{T}$ helper type $2 \mathrm{CD} 4^{+}$ T cells) are rendered resistant to killing by cytolytic T lymphocytes ${ }^{10}$. This effect is mediated by upregulation in the DC of a specific serum protease inhibitor (Spi-6) that inhibits the function of granzyme B.

There are also many unresolved issues about the development and function of memory $\mathrm{T}_{\mathrm{FH}}$ cells. $\mathrm{CD} 4^{+} \mathrm{CXCR}^{+} \mathrm{T}$ cells are also found in the circulation ${ }^{4,11}$. So what leads to the retention of some $\mathrm{T}_{\mathrm{FH}}$ cells in lymphoid tissue and the release of other $\mathrm{T}_{\mathrm{FH}}$ cells to the blood? In particular, the precise MHC class II-positive antigen-presenting cell associated with and perhaps maintaining $\mathrm{T}_{\mathrm{FH}}$ cell memory is not known. It is not a follicular DC (which do not process antigen or express MHC class II molecules); perhaps myeloid DCs or a subset of $\mathrm{B}$ cells are responsible (Fig. 1b). The function of CD69 in the retention of memory $\mathrm{T}_{\mathrm{FH}}$ cells and the location of these memory $\mathrm{T}_{\mathrm{FH}}$ cells in the node also must be elucidated.

Many issues raised by both reports are relevant to vaccination studies. Can adjuvants be selected for that will enhance beneficial effects such as the development of $\mathrm{T}_{\mathrm{FH}}$ cells but avoid unwanted effects such as DC killing?
How should booster vaccinations be administered without inadvertent promotion of negative feedback and downregulation of specific immunity? In addition, in vaccination studies, peripheral blood is typically monitored for the presence of high-affinity $\mathrm{T}$ cells after vaccination. But if a large proportion of these cells remain localized or are recruited to lymph nodes draining inoculation sites, how will this affect the assessment of vaccine efficacy? These issues must be considered in the design of future vaccine studies in animal models and in human trials.

\section{COMPETING INTERESTS STATEMENT}

The authors declare no competing financial interests.

1. Sallusto, F., Geginat, J. \& Lanzavecchia, A. Annu. Rev. Immunol. 22, 745-763 (2004).

2. Guarda, G. et al. Nat. Immunol. 8, 743-752 (2007).

3. Fazilleau, N. et al. Nat. Immunol. 8, 753-761 (2007).

4. Vinuesa, C.G., Tangye, S.G., Moser, B. \& Mackay, C.R. Nat. Rev. Immunol. 5, 853-865 (2005).

5. Hosmalin, A. et al. Blood 97, 2695-2701 (2001).

6. Yang, J., Huck, S.P., McHugh, R.S., Hermans, I.F. \& Ronchese, F. Proc. Natl. Acad. Sci. USA 103, 147-152 (2006).

7. Shiow, L.R. et al. Nature 440, 540-544 (2006).

8. Zammit, D.J., Turner, D.L., Klonowski, K.D., Lefrancois, L. \& Cauley, L.S. Immunity 24, 439-449 (2006).

9. Badovinac, V.P., Messingham, K.A., Jabbari, A., Haring, J.S. \& Harty, J.T. Nat. Med. 11, 748-756 (2005).

10. Medema, J.P. et al. J. Exp. Med. 194, 657-667 (2001).

11. Breitfeld, D. et al. J. Exp. Med. 192, 1545-1552 (2000).

12. Lindquist, R.L. et al. Nat. Immunol. 5, 1243-1250 (2004).

\section{Epigenetic regulation of Ifng expression}

\section{Charalampos G Spilianakis \& Richard A Flavell}

\section{Intensive characterization of the locus encoding interferon- $\gamma$ provides new insight into how proper gene expression is achieved in polarizing $\mathrm{T}$ cells.}

\begin{abstract}
E pigenetic regulation of gene expression $E_{\text {is an important mechanism that controls }}$ transcriptional activation or repression of the Ifng locus (encoding interferon- $\gamma($ IFN- $\gamma)$ ). In this issue of Nature Immunology, two groups report specific epigenetic changes that regulate the activation of Ifng during the process of the differentiation of $\mathrm{CD} 4^{+} \mathrm{T}$ helper cells into $\mathrm{T}$ helper type $1\left(\mathrm{~T}_{\mathrm{H}} 1\right)$ cells $^{1}$ and also the
\end{abstract}

Charalampos G. Spilianakis and Richard A. Flavell are in the Section of Immunobiology, Yale University School of Medicine and The Howard Hughes Medical Institute, New Haven, Connecticut 06520, USA. e-mail: richard.flavell@yale.edu silencing of Ifng in differentiating $\mathrm{T}_{\mathrm{H}} 2$ cells $^{2}$. In the comprehensive analysis presented by Schoenborn et al., specific histone-methylation patterns of chromatin surrounding Ifng in an extended region of over 100 kilobases are used to explain the permissive chromatin environment for Ifng expression in differentiated $\mathrm{T}_{\mathrm{H}} \mathrm{l}$ cells relative to the nonpermissive chromatin environment for Ifng expression in $\mathrm{T}_{\mathrm{H}} 2$ cells ${ }^{1}$. Additionally, Chang and Aune report potential epigenetic mechanisms that drive silencing of Ifng in differentiating $\mathrm{T}_{\mathrm{H}} 2$ cells $\mathrm{s}^{2}$. The dynamic nature of the epigenetic changes that drive the silencing of Ifng is dependent on two key transcriptional activators of $\mathrm{T}_{\mathrm{H}} 2$ cell differentiation: GATA-3 and STAT6.
T lymphocytes regulate the mammalian adaptive immune response. Effector $\mathrm{T}$ cells provide protection against intracellular and extracellular pathogens as well as tumor cells and enable many other effector mechanisms, including antibody production. The pathological consequences of overaggressive $\mathrm{T}$ cell response have been linked to allergy, autoimmunity and transplant rejection. Much of the activity of $\mathrm{T}$ cells is controlled transcriptionally. Two main populations of $\mathrm{T}$ lymphocytes mediate adaptive immunity and have been studied extensively to elucidate gene regulation in terms of trans-acting factors and to target the chromatin of the $T_{H} 1-$ and $T_{H} 2$-specific genes to identify the transcriptional regulatory 